\title{
Generalization of Subpixel Analysis for Hyperspectral Data With Flexibility in Spectral Similarity Measures
}

\author{
Jin Chen, Xiuping Jia, Wei Yang, and Bunkei Matsushita
}

\begin{abstract}
Several spectral unmixing techniques have been developed for subpixel mapping using hyperspectral data in the past two decades, among which the fully constrained least squares method based on the linear spectral mixture model (LSMM) has been widely accepted. However, the shortage of this method is that the Euclidean spectral distance measure is used, and therefore, it is sensitive to the magnitude of the spectra. While other spectral matching criteria are available, such as spectral angle mapping (SAM) and spectral information divergence (SID), the current unmixing algorithm is unable to be extended to these measures. In this paper, we propose a unified subpixel mapping framework that models the unmixing process as a best match of the unknown pixel's spectrum to a weighted sum of the endmembers' spectra. We introduce sequential quadratic programming to solve the nonlinear optimization problem encountered in the implementation of this framework. The main feature of this proposed method is that it is not restricted to any particular similarity measures. Experiments were conducted with both simulated and Hyperion data. The tests demonstrated the proposed framework's advantage in accommodating various spectral similarity measures and provided performance comparisons of the Euclidean distance measure with other spectral matching criteria including SAM, spectral correlation measure, and SID.
\end{abstract}

Index Terms-Constrained nonlinear optimization, sequential quadratic optimization, spectral mixture analysis, spectrum matching.

\section{INTRODUCTION}

$\mathbf{T}$ HE REFLECTED solar spectrum measured by a sensor mounted on an aircraft or satellite is the combination of the spectra of all the materials present within the instantaneous field of view (a pixel). Therefore, mixed pixels exist widely in a remotely sensed image. The spectral mixture not only results in inaccuracy for the materials' discrimination and classification

Manuscript received February 21, 2008; revised August 6, 2008 and October 13, 2008. First published April 7, 2009; current version published June 19, 2009. This work was supported by the Scientific Research Foundation through the State Key Laboratory of Remote Sensing Science, which is jointly supported by the Institute of Remote Sensing Applications, Chinese Academy of Sciences, and by Beijing Normal University, China.

J. Chen is with the College of Resources Science and Technology, Beijing Normal University, Beijing 100875, China (e-mail: chenjin@ires.cn).

$\mathrm{X}$. Jia is with the School of Information Technology and Electrical Engineering, University College, The University of New South Wales, Australian Defence Force Academy, Canberra, ACT 2600, Australia (e-mail: x-jia@adfa.edu.au)

W. Yang and B. Matsushita are with the Graduate School of Life and Environmental Sciences, University of Tsukuba, Tsukuba 305-8572, Japan (e-mail: yangwei1982@ires.cn; mbunkei@ sakura.cc.tsukuba.ac.jp).

Color versions of one or more of the figures in this paper are available online at http://ieeexplore.ieee.org.

Digital Object Identifier 10.1109/TGRS.2008.2011432 but also greatly hinders the development of quantitative remote sensing. In the past two decades, several unmixing techniques have been proposed including the linear spectral mixture model (LSMM) [1], neural networks [2], Gaussian mixture discriminant analysis [3], and subspace projection approach [4]. Among these methods, LSMM has been widely adopted in applications such as urban environment monitoring [5], [6], water turbidity monitoring [7], land-cover change detection [8], and vegetation-type mapping [9].

LSMM-based methods can be implemented with or without constrains. The unconstrained method is straightforward but may produce negative abundance fractions and total abundance higher than $100 \%$ that are hard to interpret. To overcome this problem, a fully constrained least squares (FCLS) method was proposed to impose the abundance sum-to-one constraint and abundance nonnegative constraint [10]. The weakness of the algorithm is that it decomposes a mixed spectrum into a weighted sum of the endmembers' brightnesses at each waveband. In other words, only the minimum spectral distance can be used as a similarity measure in implementing FCLS.

As we know, minimum spectral distance is one of the several spectral matching criteria. The shortage of this method is that it is sensitive to the absolute magnitude of the spectra. Due to external factors, such as atmospheric effect, environmental radiation, shading, etc., the magnitude of the spectrum varies greatly compared with laboratory measurements, so the brightnessbased FCLS method may produce significant errors. Other spectral matching criteria are available, such as spectral angle mapping (SAM) [11], spectral correlation measure (SCM) [12], and spectral information divergence (SID) [13]; they are less sensitive to brightness. Unfortunately, the current FCLS algorithm is unable to be extended to these similarity measures.

In this paper, we propose a generalized spectral unmixing method and introduce sequential quadratic programming (SQP) to solve the nonlinear optimization problem encountered. Under this framework, various similarity measures can be applied, including the minimum distance measure. The developed program is then tested with both simulated and EO-1 Hyperion data.

\section{MethoD}

\section{A. $L S M M$}

LSMM is reasonable when multiscattering is believed to be negligible. In LSMM, the reflectance of a pixel in the $n$th 
band $s_{n}, n=1,2, \ldots, N$, is regarded as a weighted sum of the reflectance of each endmember within a pixel

$$
s_{n}=\sum_{m=1}^{M} f_{m} a_{m, n}+e_{n}, \quad n=1,2, \ldots, N
$$

where $a_{m, n}$ is the reflectance of endmember $m$ at band $n$, $f_{m}$ is the fraction of the endmember, $M$ is the number of endmembers, and $e_{n}$ is the residual error. The fractions of the endmembers are commonly constrained by

$$
\sum_{m=1}^{M} f_{m}=1 \quad 0 \leq f_{m} \leq 1
$$

Model fit is conventionally assessed by the root-mean-square error (rmse)

$$
\text { rmse }=\sqrt{\frac{\sum_{n=1}^{N} e_{n}^{2}}{N}} .
$$

This model unmixes the spectrum based on its absolute brightness values. Unfortunately, it cannot be used to implement other similarity measures. Considering the limitation of minimum brightness distance measure, it is important to develop spectral unmixing methods using other spectral matching criteria, such as spectral correlation.

\section{B. Generalized Spectral Unmixing Framework}

Instead of decomposing a mixed pixel's brightness into a weighted sum of the endmembers' brightnesses, we propose to seek the best match of the unknown pixel spectrum to a weighted sum of the endmembers' spectra, where the user determines the matching criterion. The details are described hereinafter.

Suppose that there are $M$ endmembers' spectral vectors, $\mathbf{a}_{m}, m=1,2, \ldots, M$, obtained from images or spectral library. The reference (mixed) spectral vector $\mathbf{s}_{r}$ can be expressed as $\mathbf{s}_{r}=\sum_{m=1}^{M} f_{m} \mathbf{a}_{m}$; then, the best match of this reference spectrum to the target spectrum $\mathrm{s}$ is resolved by finding the right fractions, $f_{1}, f_{2}, \ldots, f_{M}$. These fractions of endmembers are the unmixing results for the unknown spectrum $\mathrm{s}$. Therefore, the unmixing framework can be described as follows:

$$
\begin{array}{ll}
\min & d\left(\mathbf{s}, \mathbf{s}_{r}\right)=G\left(f_{1}, f_{2}, \ldots, f_{M}\right) \\
\text { s.t. } & \sum_{m=1}^{M} f_{m}=1 \quad 0 \leq f_{m} \leq 1
\end{array}
$$

where $G$ is the objective function, which is the spectral matching criterion selected to measure the difference between the reference spectrum and the target spectrum, and the fractions of the endmembers, $f_{1}, f_{2}, \ldots, f_{M}$, are the decision variables. The constraints are the same as (2). Equation (4) represents a constrained nonlinear optimization problem, which can be solved by using the SQP method.

\section{C. $S Q P$}

The SQP method is one of the most successful methods for the numerical solution of general constrained nonlinear optimization problems. SQP is characterized by solving a sequence of quadratic programming (QP) subproblems, and the solution of QP is used as the search direction of line search in the next iteration [14].

To solve the specific optimization problem [(4)] of this paper, the constraints in (4) can be rewritten as

$$
A \mathbf{f} \geq B
$$

where

$$
A=\left[\begin{array}{rrrr}
1 & 1 & \ldots & 1 \\
-1 & -1 & \ldots & -1 \\
1 & 0 & \ldots & 0 \\
0 & 1 & \ldots & 0 \\
\ldots & \ldots & \ldots & \ldots \\
0 & 0 & \ldots & 1 \\
-1 & 0 & \ldots & 0 \\
0 & -1 & \ldots & 0 \\
\ldots & \ldots & \ldots & \ldots \\
0 & 0 & \ldots & -1
\end{array}\right] \quad B=\left[\begin{array}{r}
1 \\
-1 \\
0 \\
0 \\
\ldots \\
0 \\
-1 \\
-1 \\
\ldots \\
-1
\end{array}\right] \quad \mathbf{f}=\left[\begin{array}{c}
f_{1} \\
f_{2} \\
\cdot \\
. \\
. \\
f_{M}
\end{array}\right]
$$

$G(\mathbf{f})$ can be replaced by its local quadratic approximation at current iteration $k$

$$
\begin{aligned}
G(\mathbf{f})=G\left(\mathbf{f}^{(k)}\right)+\nabla G & \left(\mathbf{f}^{(k)}\right)^{\mathrm{T}}\left(\mathbf{f}-\mathbf{f}^{(k)}\right) \\
+ & \frac{1}{2}\left(\mathbf{f}-\mathbf{f}^{(k)}\right)^{\mathrm{T}} H_{k}\left(\mathbf{f}-\mathbf{f}^{(k)}\right)
\end{aligned}
$$

where $H_{k}$ is the Hesse matrix of $G(\mathbf{f})$ at $\mathbf{f}^{(k)}$. Then, we get an approximate QP subproblem at iteration $k$

$$
\begin{aligned}
& \min \quad Q\left(\mathbf{f}-\mathbf{f}^{(k)}\right)= \frac{1}{2}\left(\mathbf{f}-\mathbf{f}^{(k)}\right) H_{k}\left(\mathbf{f}-\mathbf{f}^{(k)}\right) \\
&+\left(\mathbf{f}-\mathbf{f}^{(k)}\right)^{\mathrm{T}} \nabla G\left(\mathbf{f}^{(k)}\right) \\
& \text { s.t. } \quad \mathbf{a}_{i}^{\mathrm{T}}\left(\mathbf{f}-\mathbf{f}^{(k)}\right) \geq 0, \quad i \in\left\{\mathbf{a}_{i}^{\mathrm{T}} \mathbf{f}^{(k)}=\mathbf{b}_{i}, \quad i=1,2, \ldots, M\right\} \\
&\left|f_{i}-f_{i}^{(k)}\right| \leq \delta, \quad i=1,2, \ldots, M
\end{aligned}
$$

where $\mathbf{a}_{i}$ and $\mathbf{b}_{i}$ are the $i$ th row vectors of matrices $A$ and $B$, respectively. $\delta$ is the user-defined convergence threshold to stop the iteration. The aforementioned equation is the quadratic approximation of the original program (4) at point $\mathbf{f}^{(k)}$, and its optimal solution is used as the search direction in the next iteration. The more detailed steps of SQP used in this paper can be found in [15] and [16].

\section{Advantages of the Generalized Spectral Unmixing Framework}

The proposed spectral unmixing framework is developed by replacing the spectral decomposing problem into matching an unknown spectrum with a mixed spectrum of the endmembers. 


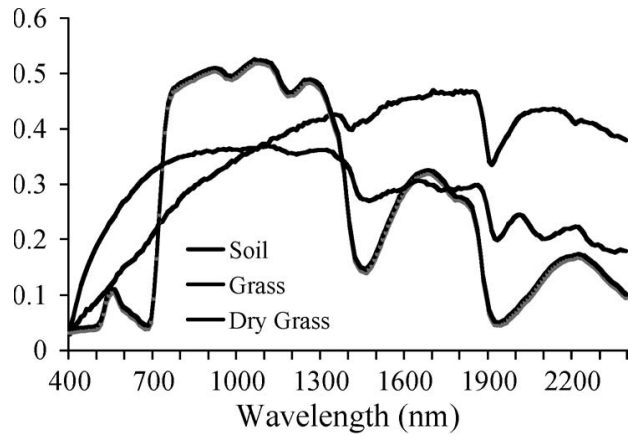

Fig. 1. Reflectance spectra of soil, grass, and dry grass.

This is a unified spectral unmixing approach, and its advantage is the ability to accommodate any spectral similarity measure that the user decides to use. The conventional spectral unmixing method given in (1) (the Euclidean-distance-based FCLS) can be implemented using this framework by selecting the objective function $G$ as the brightness difference between the reference spectrum and the target spectrum, i.e.,

$$
G=G_{\mathrm{FCLS}}=\left(\mathbf{s}-\mathbf{s}_{r}\right)^{\mathrm{T}}\left(\mathbf{s}-\mathbf{s}_{r}\right) .
$$

Unlike the model given in (1), other spectral matching algorithms can also be implemented easily with this new framework by using the selected spectral similarity measure as the objective function. For SCM, $G$ becomes

$$
G_{\mathrm{SCM}}=1-\frac{\left(\mathbf{s}_{r}-\overline{\mathbf{s}}_{r}\right)(\mathbf{s}-\overline{\mathbf{s}})}{\left\|\mathbf{s}_{r}-\overline{\mathbf{s}}_{r}\right\| \cdot\|\mathbf{s}-\overline{\mathbf{s}}\|} .
$$

The objective function $G$ for SAM is given as

$$
G_{\mathrm{SAM}}=\cos ^{-1}\left(\frac{\mathbf{s}_{r} \mathbf{s}}{\left\|\mathbf{s}_{r}\right\| \cdot\|\mathbf{s}\|}\right) .
$$

The objective function $G$ for SID is defined as

$$
G_{\mathrm{SID}}=\sum_{n=1}^{N} p_{n} \log \left(\frac{p_{n}}{q_{n}}\right)+\sum_{n=1}^{N} q_{n} \log \left(\frac{q_{n}}{p_{n}}\right)
$$

where $p_{n}$ and $q_{n}, n=1,2, \ldots, N$, the two probability mass functions produced by the reference spectrum and the target spectrum, respectively, are defined by

$$
p_{n}=\frac{s_{r, n}}{\sum_{n=1}^{N} s_{r, n}} \quad q_{n}=\frac{s_{n}}{\sum_{n=1}^{N} s_{n}} .
$$

SID is a measure of the discrepancy between the two spectra based on their corresponding probability mass functions.

\section{Algorithm Tests With Simulated Data}

Three distinct reflectance spectra of soil (grayish brown loam), grass, and dry grass were selected from the spectral library in ENVI4.3 (Fig. 1). Because the range of wavelength and spectral resolution of the original spectra were different, they were convolved to the spectral range of 400-2400 nm with resolution of $10 \mathrm{~nm}$ using the bilinear interpolation method.
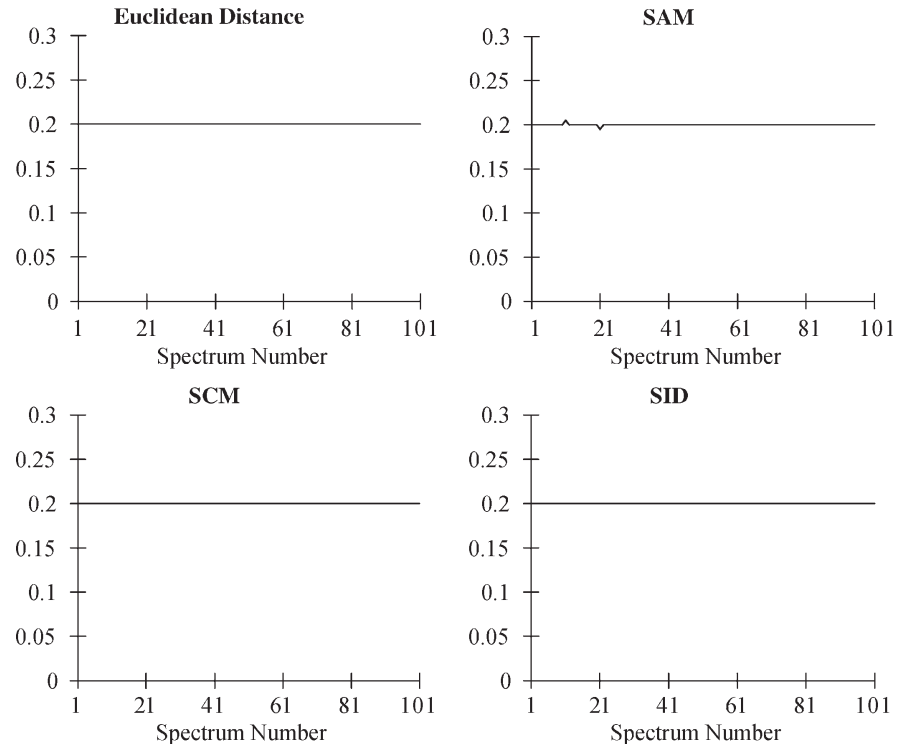

Fig. 2. Estimated fraction of grass for the Group I data.

Three groups of simulated data were generated, as detailed in the following.

Group I: The abundance of grass was fixed at $20 \%$. The abundance of dry grass decreased from $80 \%$ to $0 \%$ at $0.8 \%$ intervals. The abundance of soil increased from $0 \%$ to $80 \%$ at $0.8 \%$ intervals. Some 101 mixed spectra were generated in this way.

Group II: White Gaussian noise was added to each spectrum in Group I to achieve a 30:1 signal-to-noise ratio (SNR).

Group III: Each mixed spectrum of Group I was multiplied by a random number between 0.8 and 1.2 to produce another 101 spectra with the same spectral shape as that in Group I but different magnitude.

Four spectral similarity measures, i.e., brightness-based spectral distance, SAM, SCM, and SID, were implemented using the developed spectral unmixing framework. The performance of each method is assessed using the rmse in its estimations of the fraction of grass. The true fraction was $20 \%$, so the rmse is calculated as

$$
\text { rmse }=100 \times \sqrt{\frac{\sum_{k=1}^{101}\left(f_{\text {grass }, k}-20 \%\right)^{2}}{101}}(\%) .
$$

The estimated fractions using the four similarity measures for each group data are shown in Figs. 2-4, respectively. Table I shows the rmse of each method on the three groups of data.

It can be seen that all four methods worked very well for the Group I data, which were noise free. For the data of Group II in which small amount of white Gaussian noise was added to each spectrum, performances of the four methods were all satisfactory with quite small rmse ranging from $0.83 \%$ to $1.25 \%$. For the spectra in Group III, whose shape features are identical to the spectra in Group I but the magnitudes change significantly, the output of the brightness-based unmixing method contained significant error from the true fraction, 

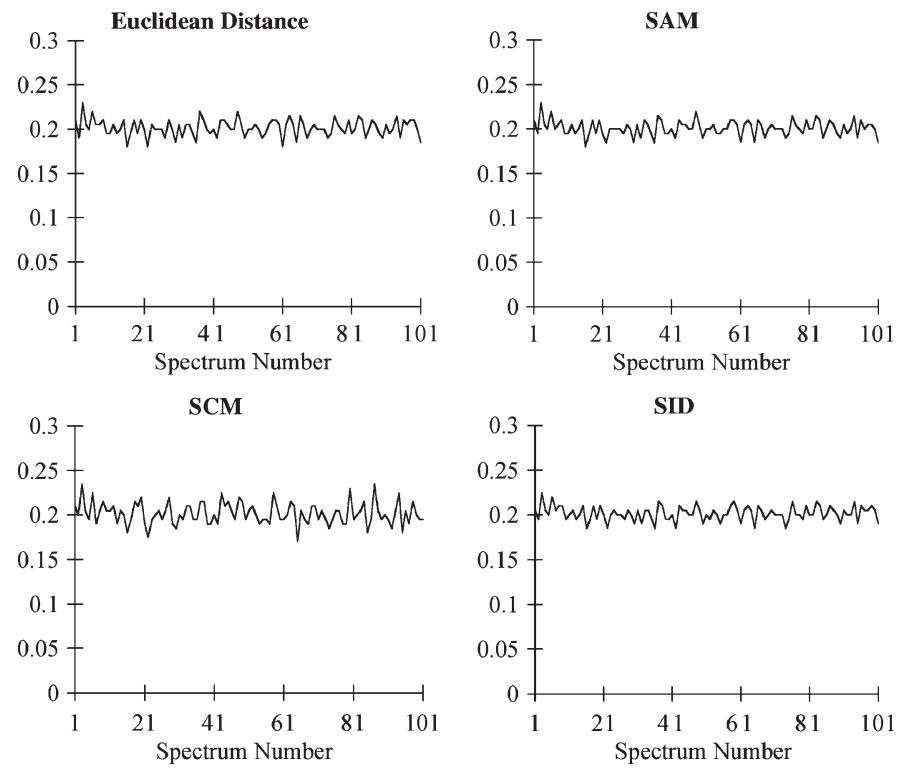

Fig. 3. Estimated fraction of grass for the Group II data.
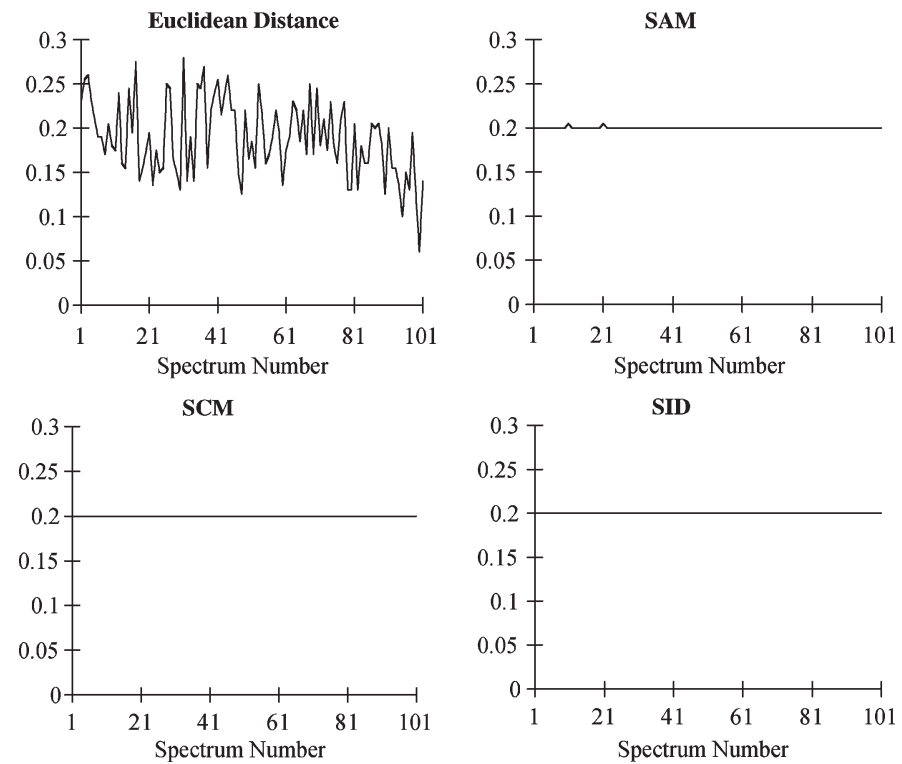

Fig. 4. Estimated fraction of grass for the Group III data.

with the largest rmse being $4.49 \%$, while the other three shapebased methods, namely, SAM, SCM, and SID, coped well with the magnitude variations, as expected (Fig. 4).

\section{Application: Subpixel Mapping of Biological SOIL CRUSTS USING HYPERION DATA}

Biological soil crusts are communities of mosses, lichens, liverworts, algae, fungi, cyanobacteria, and bacteria. They have strong effect on the functioning of the desert ecosystem and have been recommended as the top management priority in desert regions [17]. As preliminary work to protect biological soil crusts in desert regions, it is critical to identify its distribution quantitatively. Hyperion data are tested for this application and discussed in the following.
TABLE I

RMSE OF EACH UNMIXING METHOD

\begin{tabular}{ccccc}
\hline & $\begin{array}{c}\text { Euclidean } \\
\text { Distance }\end{array}$ & SAM & SCM & SID \\
\hline Group I & 0.00 & 0.07 & 0.00 & 0.00 \\
Group II & 0.94 & 0.87 & 1.25 & 0.83 \\
Group III & 4.49 & 0.07 & 0.00 & 0.00 \\
\hline
\end{tabular}

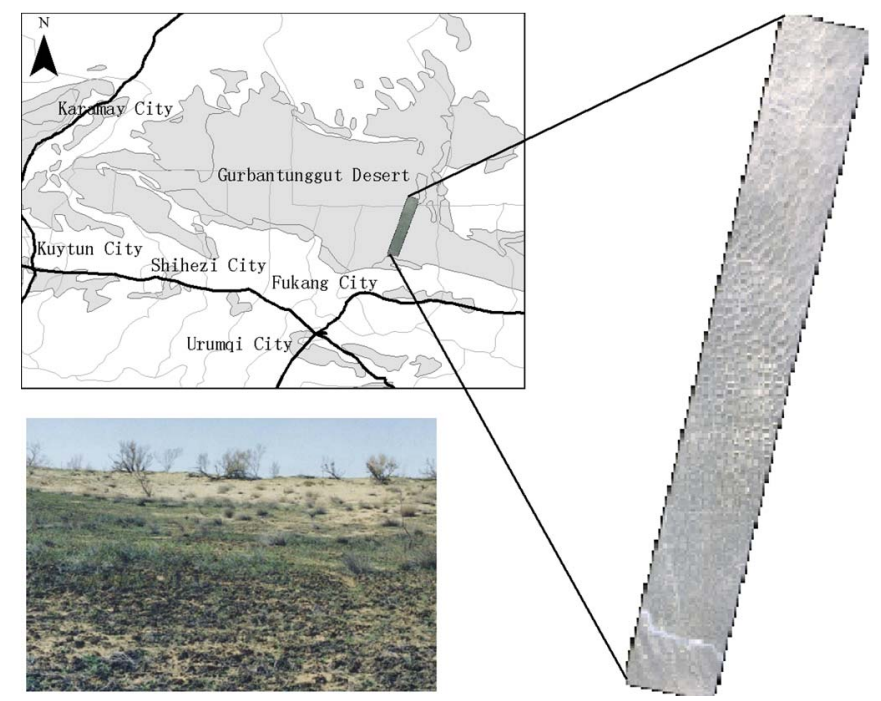

Fig. 5. Gurbantunggut Desert, Xinjiang, China, and landscape of biological soil crust.

\section{A. Study Area and Data Processing}

The study area is located in Gurbantunggut Desert $\left(44^{\circ} 11^{\prime}-46^{\circ} 20^{\prime} \mathrm{N}, 84^{\circ} 31^{\prime}-90^{\circ} 00^{\prime}\right.$ E, Fig. 5), Xinjiang, which is the second largest desert of China, with an area of $48800 \mathrm{~km}^{2}$. The area is covered by huge and dense semifixed sand dunes with stable moisture content. There are abundant lichen-dominated biological soil crusts on the sand surface of the desert, which grow most during wet and cool periods (fall and early spring) when dew, fog, or temporary rainfalls, as moisture sources, are available to species relating to the formation of biological soil crusts. Intensive fieldwork was carried out on October 20-30, 2003, during the growth peak of biological soil crusts in the desert. At that time, it was observed that annual plants died, and shrub perennials dried out. Samples of the most common land-cover types, including lichen-dominated biological soil crusts, bare sand, plant litter, and dry desert shrubs, were collected at 17 sampling sites. Three fundamental classes, i.e., lichen-dominated biological soil crusts, dry plant, and bare sand, were selected as endmembers. Their spectra were measured in the laboratory using an Analytical Spectral Devices FieldSpec Pro FR portable spectrometer (Fig. 6) [18].

The EO-1 Hyperion data of the study area were collected on October 22, 2003, when the field survey was carried out. The north portion of the Hyperion scene $(7.7 \mathrm{~km} \times 64 \mathrm{~km})$ covered by well-developed biological soil crust was selected for algorithm validation. The radiometrically calibrated Hyperion data consist of 198 bands from 400 to $2600 \mathrm{~nm}$ with spatial resolution of $30 \mathrm{~m}$ at nadir [19]. They were converted into surface 


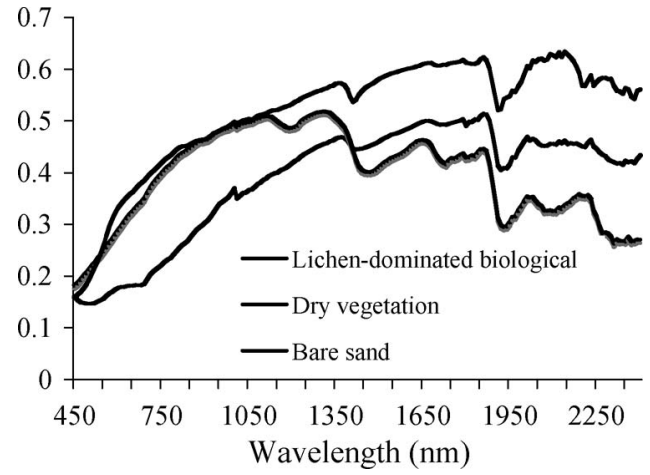

Fig. 6. Spectra of endmembers collected in Gurbantunggut Desert.

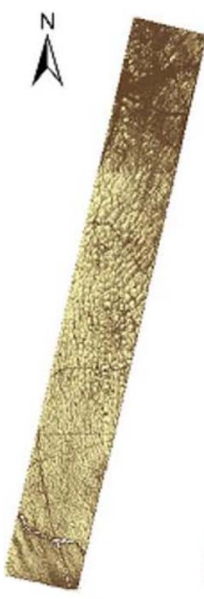

(a)

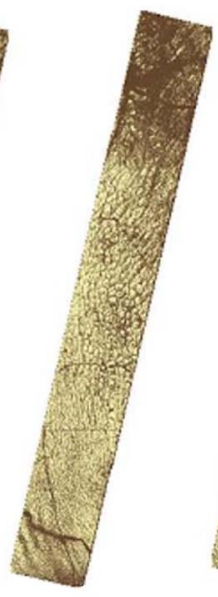

(b)

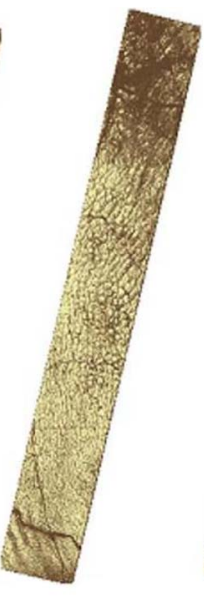

(c)

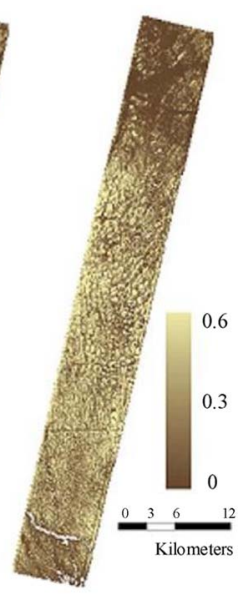

(d)
Fig. 7. Fraction of lichen-dominated biological soil crust estimated by SAM, SCM, SID, and Euclidean distance.

reflectance, using the Atmospheric Correction Now software in which a MODTRAN4-based technique was used to minimize scattering and absorption effects of several atmosphere constituents (e.g., water vapor, carbon dioxide, ozone, and oxygen). Due to the low SNR ratio at both spectral ends $(<500$ and $>2400 \mathrm{~nm})$ and heavy water absorption centered at 1400 and $1900 \mathrm{~nm}$, a total of 31 bands were removed from the original 198 bands, leaving 167 bands for use in the following experiments.

\section{B. Spectral Unmixing Results}

The conventional Euclidean-distance-based FCLS method and developed spectral unmixing methods based on SAM, SCM, and SID were applied to EO-1 Hyperion data. Fig. 7(a)-(d) shows the coverage of the lichen-dominated biological soil crusts obtained using the four difference similarity measures, respectively. We can see that the spatial distribution pattern of the four results is consistent in most of areas with about $50 \%$ abundance of biological soil crusts in the center of the image, patchy in the south, and low abundance in the north.

In order to assess the performance of each similarity measure quantitatively, a random sampling method was applied. For classification accuracy validation, there is a consensus among researchers that a minimum of 50 samples for each category

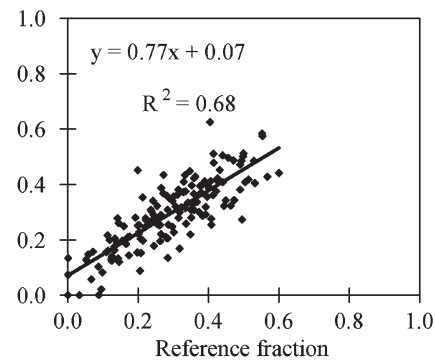

(a)

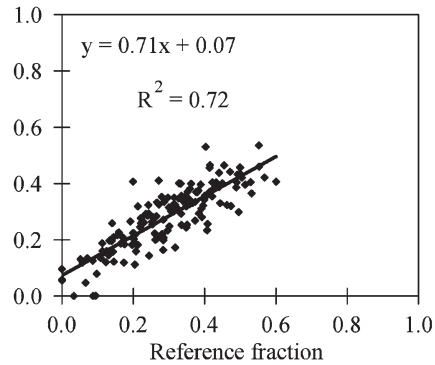

(c)

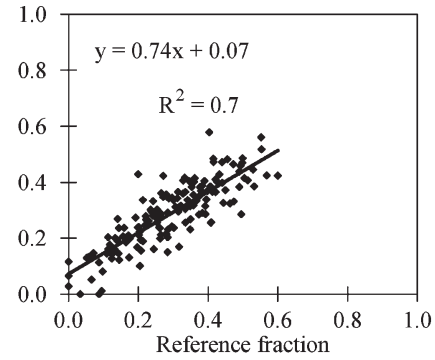

(b)

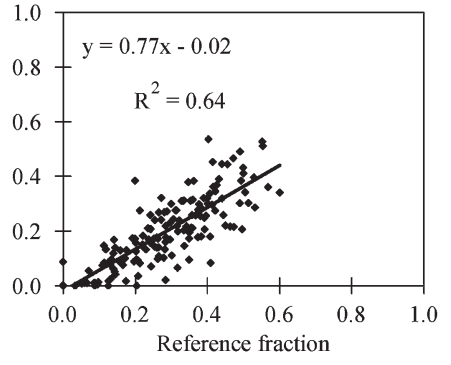

(d)
Fig. 8. Accuracy comparisons of the lichen-dominated biological soil crusts derived from the unmixing methods based on (a) SAM, (b) SCM, (c) SID, and (d) Euclidean distance.

is reasonable from both statistical and practical point of views with random or stratified random sampling schemes [20]. Since there are three endmembers in this paper, 150 sites of coverage for lichen-dominated biological soil crust were selected for the field survey, and the locations of these sites were recorded by GPS. A mean abundance within the $3 \times 3$ sampling unit $(30 \mathrm{~m})$ around the GPS point was utilized for accuracy assessment to reduce the impacts of geometric errors associated with Hyperion and GPS. Comparisons between the reference fraction (observed fraction) $f_{o}$ and estimated fraction $f$ are shown in Fig. 8. Two indexes of error measurement, rmse, and systematic error (SE) were utilized in this paper to evaluate the accuracy of results derived from the unmixing methods based on spectral Euclidean distance, SAM, SCM, and SID

$$
\begin{aligned}
\mathrm{rmse} & =100 \times \sqrt{\frac{\sum_{k=1}^{K}\left(f_{k}-f_{o, k}\right)^{2}}{K}}(\%) \\
\mathrm{SE} & =100 \times \frac{\sum_{k}^{K}\left(f_{k}-f_{o, k}\right)}{K}(\%) .
\end{aligned}
$$

The correlation between estimated fractions and reference fractions was also used to evaluate the performance. The "goodness of fit" of the correlation was assessed by the slope, intercept, and $R$-squared values, where, in an ideal case, the slope of the relationship would equal to one, the intercept is zero, and the $R$-squared value approaches one (Table II).

It can be seen that both rmse and SE produced by SAM, SCM, and SID were all much smaller than that by Euclidean distance. The SEs of SCM, SID, and Euclidean distance were negative, which indicated that the coverage of lichen-dominated biological soil crusts was underestimated, while the result of SAM overestimated with the positive SE. In the correlation 
TABLE II

COMPARISONS OF LICHEN-DOMINATED BIOLOGICAL SOIL CRUST Estimation ACCURACY

\begin{tabular}{cccccc}
\hline & RMSE & SE & Slope & Intercept & $\mathrm{R}^{2}$ \\
\hline SAM & 7.65 & 0.32 & 0.77 & 0.07 & 0.68 \\
SCM & 7.25 & -0.49 & 0.74 & 0.07 & 0.7 \\
SID & 7.16 & -1.31 & 0.71 & 0.07 & 0.72 \\
Euclidean Distance & 12.02 & -8.76 & 0.77 & -0.02 & 0.64 \\
\hline
\end{tabular}

analysis between observed and estimated fractions, the $R^{2}$ values of the shape-based similar measure methods (SAM, SCM, and SID) were all higher than that of the magnitudebased method. All these results demonstrated that the SAM, SCM, and SID measures outperformed the Euclidean distance in detecting biological soil crusts using Hyperion data. As we know, the SNR of EO-1 Hyperion data is not high which can reduce the accuracy of the reflectance derived from DN values. The accurate atmospheric parameters at the time that the data were recorded are often unavailable; therefore, some errors will be included in the atmospheric correction procedure. The aforementioned two points make the shape-based spectral unmixing method more suitable than the brightnessbased technique. Among the three shape-based measures, their performances are similar in terms of rmse and SE. However, the $R^{2}$ of the SID method was the higher than that of SAM and SCM. In other words, this set of experiments showed that SID is the most effective method among the shape-based methods, which is consistent with the conclusion presented in [21].

\section{DiscusSION AND CONCLUSION}

In this paper, a generalized spectral unmixing framework was developed, in which various spectral similarity measures can be applied. Three shape-based spectral matching criteria, i.e., SAM, SCM, and SID, and spectral brightness-based methods using the Euclidean distance measure were investigated using both simulated and EO-1 Hyperion data sets. The experimental results suggest that the methods based on SAM, SCM, and SID perform better than the method based on Euclidean distance when the shape feature of the spectra keeps well while the magnitude changes significantly, and SID is more stable than SAM and SCM.

The flexibility of the developed subpixel mapping framework in the selection of spectral similarity measures removes the limitation that the spectral unmixing has to be achieved by decomposing an unknown spectrum's brightness into a weighted sum of the brightness of each endmember. The spectral-shapebased measures are less sensitive to magnitude variations. For example, SAM is independent of solar illumination factors and can cope with the pixels in the shade. Moreover, shape-based methods have lower requirement for exact atmospheric correction than the brightness-based algorithm, which can avoid the difficulties in obtaining all the atmospheric parameters.

The developed spectral unmixing framework searches the best match of the unknown pixel spectrum to a weighted sum of the endmembers' spectra. It is anticipated that the noise contained in the measured spectrum may have a lower impact on the unmixing process. This will be investigated as future work. Further improvement also includes efficient algorithm development to solve the constrained nonlinear optimization problem so that the spectral unmixing process can be sped up.

\section{ACKNOWLEDGMENT}

The authors would like to thank the support by the Scientific Research Foundation for the Returned Overseas Chinese Scholars, State Education Ministry, China.

\section{REFERENCES}

[1] J. B. Adams, M. O. Smith, and P. E. Johnson, "Spectral mixture modeling: A new analysis of rock and soil types at the Viking Lander I site," $J$. Geophys. Res., vol. 91, pp. 8098-8112, Jul. 1985.

[2] P. M. Atkinson, M. E. J. Cutler, and H. Lewis, "Mapping sub-pixel proportional land cover with AVHRR imagery," Int. J. Remote Sens., vol. 18, no. 4, pp. 917-935, Mar. 1997.

[3] J. Ju, E. D. Kolaczyk, and S. Gopal, "Gaussian mixture discriminant analysis and sub-pixel land cover characterization in remote sensing," Remote Sens. Environ., vol. 84, no. 4, pp. 550-560, Apr. 2003.

[4] C.-I. Chang, X. Zhao, M. L. G. Althouse, and J.-J. Pan, "Least squares subspace projection approach to mixed pixel classification in hyperspectral images," IEEE Trans. Geosci. Remote Sens., vol. 36, no. 3, pp. 898912, May 1998.

[5] Q. H. Weng, D. S. Lu, and J. Schubring, "Estimation of land surface temperature-vegetation abundance relationship for urban heat island studies," Remote Sens. Environ., vol. 89, no. 4, pp. 467-483, Feb. 2004.

[6] D. S. Lu and Q. H. Weng, "Spectral mixture analysis of ASTER images for examining the relationship between urban thermal features and biophysical descriptors in Indianapolis, Indiana, USA," Remote Sens. Environ., vol. 104, no. 2, pp. 157-167, Sep. 2006.

[7] S. Kameyama, Y. Yamagata, F. Nakamura, and M. Kaneko, "Development of WTI and turbidity estimation model using SMA-Application to Kushiro Mire, eastern Hokkaido, Japan," Remote Sens. Environ., vol. 77, no. 1, pp. 1-9, Jul. 2001.

[8] J. Rogan, J. Franklin, and D. A. Roberts, "A comparison of methods for monitoring multitemporal vegetation change using Thematic Mapper imagery," Remote Sens. Environ., vol. 80, no. 1, pp. 143-156, Apr. 2000.

[9] D. Riano, E. Chuvieco, S. Ustin, R. Zomer, P. Dennison, D. Roberts, and J. Salas, "Assessment of vegetation regeneration after fire through multitemporal analysis of AVIRIS images in the Santa Monica Mountains," Remote Sens. Environ., vol. 79, no. 1, pp. 60-71, Jan. 2002.

[10] D. C. Heinz and C.-I. Chang, "Fully constrained least squares linear spectral mixture analysis method for material quantification in hyperspectral imagery," IEEE Trans. Geosci. Remote Sens., vol. 39, no. 3, pp. 529-545, Mar. 2001.

[11] F. A. Kruse, A. B. Lefkoff, J. W. Boardman, K. B. Heidebrecht, A. T. Shapiro, P. J. Barloon, and A. F. H. Goetz, "The spectral image processing system (SIPS)-Interactive visualization and analysis of imaging spectrometer data," Remote Sens. Environ., vol. 44, no. 2/3, pp. 145-163, May/Jun. 1993.

[12] F. van der Meer and W. Bakker, "Cross correlogram spectral matching (CCSM): Application to surface mineralogical mapping using AVIRIS data from Cuprite, Nevada," Remote Sens. Environ., vol. 61, no. 3, pp. 371-382, Sep. 1997.

[13] C.-I. Chang, "An information theoretic-based approach to spectral variability, similarity and discriminability for hyperspectral image analysis," IEEE Trans. Inf. Theory, vol. 46, no. 5, pp. 1927-1932, Aug. 2000.

[14] K. Schittkowski, "The nonlinear programming method of Wilson, Han, and Powell with an augmented Lagrangian type line search functionPart 2: An efficient implementation with linear least squares subproblems," Numer. Math., vol. 38, no. 1, pp. 115-127, Feb. 1981.

[15] G. C. Zhang, W. J. Wang, H. L. Han, and L. Zhang, Computational Methods for Nonlinear Optimization. Beijing, China: Higher Educ., 2005. (in Chinese).

[16] N. S. Bazaraa and C. M. Shetty, Nonlinear Programming: Theory and Algorithms. New York: Wiley, 1997.

[17] J. Belnap, "The world at your feet: Desert biological soil crusts," Front. Ecol. Environ., vol. 1, no. 5, pp. 181-189, 2003. 
[18] J. Chen, M. Y. Zhang, L. Wang, H. Shimazaki, and M. Tamura, "A new index for mapping lichen-dominated biological soil crusts in desert areas," Remote Sens. Environ., vol. 96, no. 2, pp. 165-175, May 2005.

[19] B. Datt, T. R. McVicar, T. G. van Niel, D. L. B. Jupp, and J. S. Pearlman, "Preprocessing EO-1 Hyperion hyperspectral data to support the application of agricultural indexes," IEEE Trans. Geosci. Remote Sens., vol. 41, no. 6, pp. 1246-1259, Jun. 2003.

[20] R. G. Congalton, "A review of assessing the accuracy of classifications of remotely sensed data," Remote Sens. Environ., vol. 37, no. 1, pp. 35-46, Jul. 1991.

[21] F. van der Meer, "The effectiveness of spectral similarity measures for the analysis of hyperspectral imagery," Int. J. Appl. Earth Observ. Geoinf., vol. 8, no. 1, pp. 3-17, Jan. 2006.

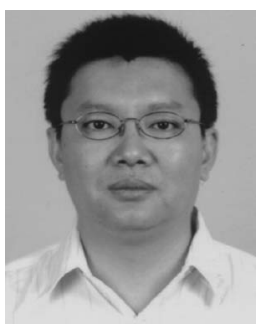

Jin Chen received the B.A. and M.S. degrees in geography from Beijing Normal University, Beijing, China, in 1989 and 1992, respectively, and the Ph.D. degree in civil engineering from Kyushu University, Fukuoka, Japan, in 2000.

$\mathrm{He}$ was a Postdoc with the University of California, Berkeley (2000-2001), and with the National Institute of Environmental Studies, Tsukuba, Japan (2001-2004). He is currently a Professor with the College of Resources Science and Technology, Beijing Normal University. His recent research interests include remote sensing modeling and vegetation parameter retrieval through the inversion of remote sensing models.

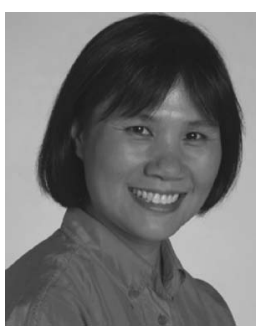

Xiuping Jia received the B.Eng. degree from the Beijing University of Posts and Telecommunications, Beijing, China, in 1982 and the Ph.D. degree in electrical engineering from The University of New South Wales, Sydney, Australia, in 1996.

Since 1988, she has been with the School of Information Technology and Electrical Engineering, University College, The University of New South Wales, Australian Defence Force Academy, Canberra, Australia, where she is currently a Senior Lecturer. She is also a Guest Professor with Beijing Normal University, Beijing. She is the coauthor of the remote sensing textbook entitled Remote Sensing Digital Image Analysis [Springer-Verlag, 3rd (1999) and 4th eds. (2006)]. Her research interests include remote sensing and imaging spectrometry.

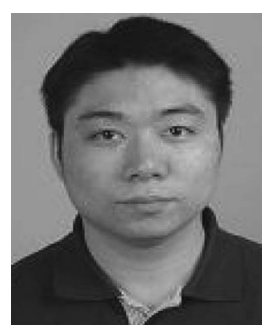

Wei Yang received the B.S. degree in physics and the M.S. degree in civil engineering from Beijing Normal University, Beijing, China, in 2005 and 2008 , respectively. He is currently working toward the Ph.D. degree in the Graduate School of Life and Environmental Sciences, University of Tsukuba, Tsukuba, Japan.

He has worked on the effect of topography to vegetation index. His current research includes spectral mixture analysis and case- 2 water remote sensing algorithms.

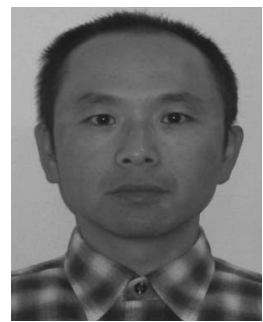

Bunkei Matsushita received the B.S. degree in information engineering from Xidian University, Xi' an, China, in 1990 and the M.S. and Ph.D. degrees in environmental earth science from Hokkaido University, Sapporo, Japan, in 1995 and 1998, respectively.

$\mathrm{He}$ is currently an Assistant Professor with the Graduate School of Life and Environmental Sciences, University of Tsukuba, Tsukuba, Japan, where he teaches and conducts research on integrating remote sensing/GIS techniques with process-based ecosystem models. His current research includes remote sensing of case-2 waters and impervious surface area of watersheds based on spectral mixture analysis. 